\title{
Coarsening of solid-liquid mixtures in a random acceleration field
}

\author{
J. Ross Thomson \\ Supercomputer Computations Research Institute, Florida State University, Tallahassee, Florida 32306-4052 \\ Jaume Casademunt \\ Departament d'Estructura i Constituents de la Materia, Universitat de Barcelona, \\ 08028 Barcelona, Spain \\ François Drolet \\ Supercomputer Computations Research Institute, Florida State University, Tallahassee, Florida 32306-4052 \\ Jorge Viñals \\ Supercomputer Computations Research Institute, Florida State University, \\ Tallahassee, Florida 32306-4052 and Department of Chemical Engineering, FAMU/FSU College of \\ Engineering, Tallahassee, Florida 32310
}

(Received 17 January 1996; accepted 2 January 1997)

\begin{abstract}
The effects of flow induced by a random acceleration field (g-jitter) are considered in two related situations that are of interest for microgravity fluid experiments: the random motion of isolated buoyant particles, and diffusion driven coarsening of a solid-liquid mixture. We start by analyzing in detail actual accelerometer data gathered during a recent microgravity mission, and obtain the values of the parameters defining a previously introduced stochastic model of this acceleration field. The diffusive motion of a single solid particle suspended in an incompressible fluid that is subjected to such random accelerations is considered, and mean squared velocities and effective diffusion coefficients are explicitly given. We next study the flow induced by an ensemble of such particles, and show the existence of a hydrodynamically induced attraction between pairs of particles at distances large compared with their radii, and repulsion at short distances. Finally, a mean field analysis is used to estimate the effect of g-jitter on diffusion controlled coarsening of a solid-liquid mixture. Corrections to classical coarsening rates due to the induced fluid motion are calculated, and estimates are given for coarsening of $\mathrm{Sn}$-rich particles in a $\mathrm{Sn}-\mathrm{Pb}$ eutectic fluid, an experiment to be conducted in microgravity in the near future. (C) 1997 American Institute of Physics.
\end{abstract}

[S1070-6631(97)00305-X]

\section{INTRODUCTION}

With recent frequent access to a microgravity environment, more attention is being paid to a precise characterization of the effective acceleration environment onboard spacecraft (g-jitter), as well as to the analysis of potential effects of such an environment on a number of experiments, compared to an ideal zero gravity situation. ${ }^{1-4}$ We study in this paper the motion induced on particles that are suspended in an incompressible fluid by an externally imposed random acceleration field. As an extension, coarsening of a solidliquid mixture is considered, and the effects of g-jitter estimated for the case of a $\mathrm{Sn}-\mathrm{Pb}$ eutectic. This system will be studied in microgravity in the near future.

Whereas qualitative information on the residual acceleration field onboard spacecraft has been available for some time, it is only recently that a systematic effort has been made to collect long temporal sequences of acceleration data over a fairly wide frequency range. ${ }^{5,6}$ In the frequency range we study $\left(10^{-1}-10^{2} \mathrm{~Hz}\right)$, the SAMS project now routinely determines for each mission the three components of the residual acceleration field at selected points in the spacecraft. This includes, in some cases, sensor heads at the same location where an experiment potentially susceptible to this kind of disturbances is being conducted.

From a theoretical point of view, the first issue to be addressed concerns the introduction of a suitable model of the residual acceleration field. Based on available accelerometer data and their associated power spectra, it seems necessary to distinguish between frequency components that can be modeled as systematic (or deterministic), and thus traced back to some mechanical device producing a periodic disturbance of known amplitude and frequency, and random components arising from a number of independent sources with variable frequencies and intensities. We further note that physical sources of accelerations contribute to the overall acceleration environment in two ways: directly, and indirectly by exciting some of the natural vibration modes of the spacecraft.

Most of the studies to date have focused on a deterministic acceleration field modeled as a superposition of periodic functions of fixed amplitudes and frequencies. ${ }^{7-11}$ Also, some studies have considered the effects of short and isolated pulses. ${ }^{9}$ The approach presented in this paper, on the other hand, models g-jitter as a random process in time. ${ }^{12,13}$ We assume that the process obeys Gaussian statistics, consistent with the assumption that many independent sources contribute to the acceleration field, thus requiring the knowledge of only the first two statistical moments of the effective acceleration field $\vec{g}(t)$. We choose $\langle\vec{g}\rangle=0$, where \langle\rangle denotes an ensemble average. In general, a nonzero average can be incorporated into a steady component, and $\vec{g}$ redefined to be the deviation from the average. We do not consider here the 
effect of this component. To model the statistical behavior of the second moment we chose a Gaussian process called narrow band noise, defined by the correlation function,

$$
\begin{aligned}
C_{i j}\left(t-t^{\prime}\right) & =\left\langle g_{i}(t) g_{j}\left(t^{\prime}\right)\right\rangle \\
& =\delta_{i j}\left\langle g^{2}\right\rangle e^{-\left|t-t^{\prime}\right| / \tau} \cos \Omega\left(t-t^{\prime}\right),
\end{aligned}
$$

where $g_{i}(t)$ is any of the three components of the acceleration field, and $\left\langle g^{2}\right\rangle, \tau$, and $\Omega$ are three constants which characterize the process: its intensity, a correlation time, and a characteristic angular frequency. A particular advantage of this process is that it allows interpolation between two well known limits: the white noise limit when $\Omega \tau \rightarrow 0$ with $\left\langle g^{2}\right\rangle \tau=D$ finite, in which no frequency component is preferred, and monochromatic noise when $\Omega \tau \rightarrow \infty,\left\langle g^{2}\right\rangle$ finite, in which each realization of the noise is a periodic function of angular frequency $\Omega$. In this case, the ensemble refers to a distribution of amplitudes and phases, with identical angular frequency for each realization. Monochromatic noise is akin to the deterministic studies in which $\vec{g}(t)$ is modeled by a periodic function, but still retains random values of the amplitude and phase.

In Sec. II, we present a statistical analysis of a long time series gathered by the SAMS team during the recent SL-J mission. A window of approximately six hours (sampled at $250 \mathrm{~Hz}$ ) is analyzed to determine the existence of deterministic and random components, and to calculate the values of the parameters needed to characterize both. A given time series can appear to be deterministic or stochastic depending on the range analyzed: If a random function is correlated over times of the order of $\tau$, its time series will appear deterministic when analyzed over time windows $T \ll \tau$, and random otherwise. In the case of the SAMS time series that we have analyzed, we find that there is a systematic or deterministic component of frequency $17 \mathrm{~Hz}$. The rest of the spectrum is comprised of a superposition of random components with small correlation time, and a white noise background (with a correlation time no longer than the sampling period of $1 / 250 \mathrm{~s}$ ). We also find significant deviations from Gaussianity, mainly in the larger amplitude impulses. It may be necessary to introduce other stochastic models that are not Gaussian to study these contributions (shot noise, for example).

Section III considers the motion of a particle suspended in an incompressible fluid of different density, when the fluid is subjected to the acceleration field described in Sec. II. If the residual acceleration field is deterministic and periodic, the suspended particle performs an oscillatory motion, with both velocity and displacement bounded. If, on the other hand, the acceleration field is random, the mean squared velocity of the particle is bounded, but its mean squared displacement grows linearly in time. The effective diffusive coefficient is given as a function of the parameters of the fluid and noise. In particular, we note that measurement of the mean squared displacement of a suspended particle in a microgravity environment would provide an independent data set from which one could infer the parameters that characterize the residual acceleration field. A similar principle has been used to design a passive accelerometer system ${ }^{14}$ to ob-

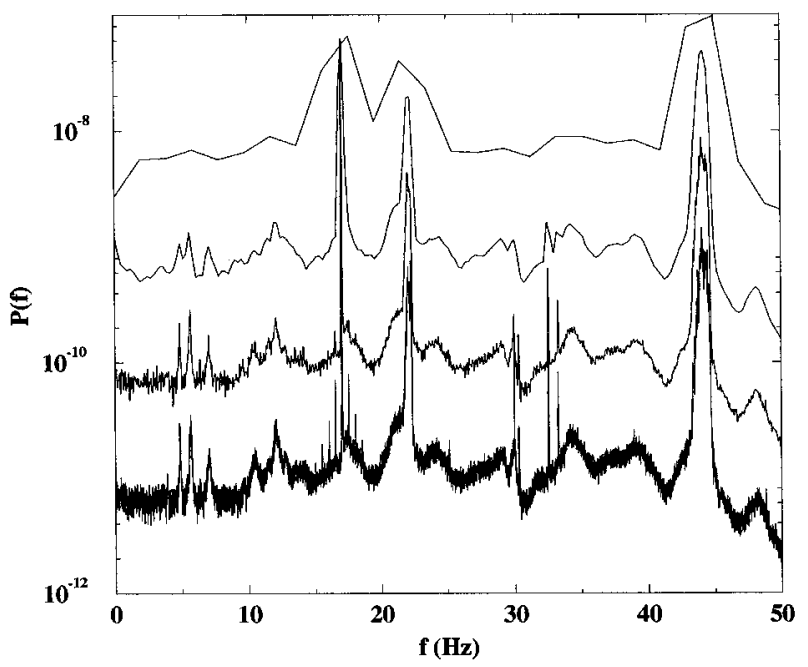

FIG. 1. Power spectrum as a function of frequency averaged over a six hour interval during the SL-J mission. The curves shown correspond to spectra calculated over windows of size (from top to bottom) $N=64,512,4096$, and 32768 . The amplitude of the peak at $f=17 \mathrm{~Hz}$ is independent of $N$, whereas the amplitude of the peaks at both $22 \mathrm{~Hz}$ and $44 \mathrm{~Hz}$ decreases with $N$. Also in this latter case, the shape of the peaks is independent of $N$.

tain the steady component of the residual acceleration field from the linear drift of a suspended particle. Not surprisingly, it has proved difficult in actual microgravity conditions to maintain a well defined alignment of the container with respect to the residual acceleration field.

Section III also addresses the effects of g-jitter on coarsening of a solid-liquid mixture. In principle, a random acceleration field may induce a random velocity field in the fluid that may lead in turn to enhanced coalescence and solute transport, and therefore introduce deviations from purely diffusion controlled coarsening. The analysis focuses on the solid-liquid mixture $\mathrm{Pb}-\mathrm{Sn}$ which will be studied in microgravity in the near future. We find that g-jitter effects are small for the conditions of the experiment, and with the values of the noise found in Sec. II.

\section{TIME SERIES ANALYSIS OF g-JITTER DURING THE SL-J MISSION}

The analysis described in this section is based on actual g-jitter data collected during the SL-J mission (SAMS-258) that flew on September 13-20, 1993. We have focused on the head A SAMS detector, and studied the series during the time window MET 0017 to MET 0023, roughly a period of six hours. All three Cartesian components of the residual acceleration field have been included in the analysis. The sampling frequency is $250 \mathrm{~Hz}$. The data used was gathered continuously throughout the period mentioned, with automatic re-calibration of the sensor heads when needed (corrections for the signal gain have been taken into account according to the calibration data also gathered during the mission).

We do not focus here on some basic statistical properties of the signal which are already automatically monitored (its running mean and root mean square values), but address two 
basic points: (a) the existence of deterministic and random components during this particular observation period, and (b) the Gaussian nature of the time series.

Consider first a temporal series $g(t)$ and its power spectrum over a finite window $[-T, T]$ defined by

$$
P_{T}(n)=\frac{1}{2 T} \int_{-T}^{T} C(t) e^{-i n \pi t / T} d t
$$

where $C(t)$ is the autocorrelation function defined in Eq. (1). The autocorrelation function can be obtained from $P_{T}(n)$ as a Fourier series,

$$
C_{T}(t)=\sum_{n=-\infty}^{\infty} P_{T}(n) e^{i n \pi t / T},
$$

where we have introduced the notation $C_{T}(t)$ to indicate that $C_{T}(t)=C(t)$ in $[-T, T]$, and is periodic outside of this interval. The integral in Eq. (2) can be evaluated explicitly to yield

$$
\begin{aligned}
P_{T}(n)= & \frac{\left\langle g^{2}\right\rangle}{2 T}\left[\frac{e^{-\lambda T}\left(-\lambda \cos \left(\frac{n \pi}{T}-\Omega\right) T+\left(\frac{n \pi}{T}-\Omega\right) \sin \left(\frac{n \pi}{T}-\Omega\right) T\right)+\lambda}{\lambda^{2}+\left(\frac{n \pi}{T}-\Omega\right)^{2}}\right. \\
& \left.+\frac{e^{-\lambda T}\left(-\lambda \cos \left(\frac{n \pi}{T}+\Omega\right) T+\left(\frac{n \pi}{T}+\Omega\right) \sin \left(\frac{n \pi}{T}+\Omega\right) T\right)+\lambda}{\lambda^{2}+\left(\frac{n \pi}{T}+\Omega\right)^{2}}\right],
\end{aligned}
$$

where $\lambda=1 / \tau$. In the white noise limit $P_{T}(n)=D / T$, whereas in the monochromatic limit $P_{T}(n)=\left(\left\langle g^{2}\right\rangle /\right.$ 2) $\left\{\delta_{n \pi / T, \Omega}+\delta_{n \pi / T,-\Omega}\right\}$. In the first case, the dominant contribution comes from the term $1 / \tau$ in Eq. (4), whereas in the second case it comes from the term proportional to $\sin [(n \pi / T)-\Omega] T$. The important point to notice is that in the white noise limit $P_{T}(n)$ is inversely proportional to the window size $T$, whereas in the monochromatic limit $P_{T}(n)$ is independent of $T$. Thus, we argue, an analysis of the power spectrum $P_{T}(n)$ as a function of the window size can provide information on the existence of deterministic or random contribution, at least within the available ranges of $T$ and $\tau$.

These results are in fact more generally valid and not restricted to narrow band noise. Consider the integral

$$
\hat{g}_{T}(n)=\frac{1}{2 T} \int_{-T}^{T} d t e^{-i n \pi t / T} g(t) .
$$

If $g(t)$ is a random process, with a correlation time $\tau \ll T$, then for each $n \hat{g}_{T}(n)$ is the sum of approximately $2 T / \tau$ statistically independent variables. Therefore, according to the Central Limit Theorem, the integral will obey Gaussian statistics, with variance $\mathscr{Q}(T / \tau)$. As a consequence, $\hat{g}_{T}(n) \sim \mathcal{C}[(1 / T) \sqrt{T / \tau}]=\mathcal{Q}(1 / \sqrt{T}) \quad$ or $\quad P_{T}(n)=\left|\hat{g}_{T}(n)\right|^{2}$ $\sim \mathscr{Q}(1 / T)$, in agreement with the result obtained for narrow band noise in the limit of short correlation time. On the other hand, for most deterministic functions $\hat{g}_{T}(n) \sim \mathscr{Q}(1)$ instead, and $P_{T}(n)$ is independent of the window size $T$.

We have obtained an estimate of $P_{T}(n)$ for the time series of $g(t)$ obtained during the SL-J mission and for a range of values of $T$. Since the time series is discrete, we consider windows comprising $N$ data points, with $N \Delta t$ $=2 T$ where $1 / \Delta t=250 \mathrm{~s}^{-1}$ is the sampling rate (further details on various methods to estimate power spectra can be found in Ref. 15). Briefly, the power spectrum for a stationary process, one in which its statistical properties are independent of time, is calculated by averaging $P_{T}$, also known as the periodogram. The relative statistical error associated with a single periodogram is $100 \%$ for all frequencies. Reduction in error by a factor proportional to $1 / N_{p}$ can be achieved by averaging $N_{p}$ periodograms calculated over disjoint time intervals. The estimate of the power spectrum presented here is obtained from approximately 6 hours of data, sampled at $250 \mathrm{~Hz}$. Each periodogram is calculated for a fixed number of sample points (beginning with 64 and increasing by factors of two) and then averaged over the entire 6 hour period. The resulting estimates of the power spectra are summarized in Fig. 1. The power spectrum is broadband, with a few peaks at fixed frequencies. The background intensity does decrease with increasing $N$, indicating its random nature.

To further elucidate the scaling with $T$, we show in Fig. 2 the value of $P_{T}$ at selected frequencies as a function of $T$. The frequency components shown in this figure include the peaks of Fig. 1, and one intermediate value. Three types of behavior emerge. First, the value of $P_{T}(f=17 \mathrm{~Hz})$ is independent of $T$ for the range of window lengths studied. Therefore, and within this range, this component appears to be deterministic in nature with an amplitude $\sqrt{\left\langle g^{2}\right\rangle}=3.56$ $\times 10^{-4} g_{E}$, where $g_{E}$ is the intensity of the gravitational field on the Earth's surface. There are two additional components that have a finite correlation time. We have fitted the amplitude of the peak to $\left\langle g^{2}\right\rangle \tau\left(1-e^{-T / \tau}\right) / 2 T$ 


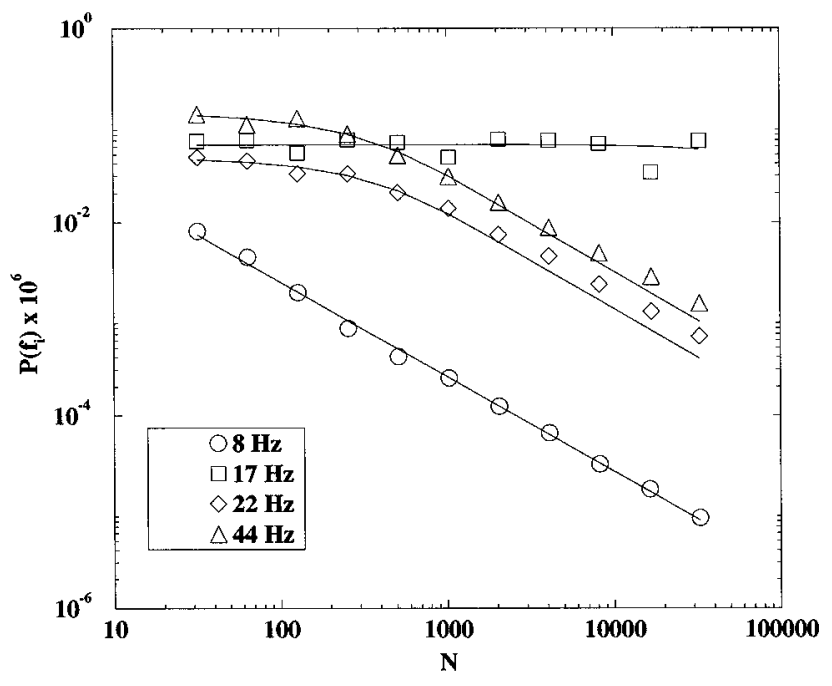

FIG. 2. Amplitude versus window size $N$ for a few selected frequencies to display their deterministic or random nature. The amplitude of the $17 \mathrm{~Hz}$ component remains independent of $N$, indicating its deterministic character for the range of window sizes analyzed. Two other components display mixed behavior, with a finite correlation time of the order of $1 \mathrm{~s}$. There is also a clear white noise background, exemplified by the amplitude of the power spectrum at $8 \mathrm{~Hz}$.

and estimated for the component at $22 \mathrm{~Hz}, \sqrt{\left\langle g^{2}\right\rangle}$ $=3.06 \times 10^{-4} g_{E}$, and $\tau=1.09 \mathrm{~s}$, whereas for $44 \mathrm{~Hz}$ we find $\sqrt{\left\langle g^{2}\right\rangle}=5.20 \times 10^{-4} g_{E}$ and $\tau=0.91 \mathrm{~s}$. As an estimate of the white noise background, we obtain from the slope of the intensity of the $8 \mathrm{~Hz}$ component versus $N$ the value $D=8.61 \times 10^{-4} \mathrm{~cm}^{2} / \mathrm{s}^{3}$.

In summary, assuming that the various frequency components can be studied independently, and that they are independent of the broadband background, we conclude that the time series analyzed contains a deterministic component (i.e., a component with a correlation time larger than the largest window studied), a few isolated components of large amplitude but small correlation time, and a fairly constant background, of smaller amplitude, and very small correlation time.

To further investigate the statistical nature of the acceleration we calculate a number of statistical moments of $g(t)$. We first present the (one-point) probability distribution of $g$ obtained from a histogram of the time series. The histogram comprises 200 bins of width $0.008 g_{E}$. The result is shown in Fig. 3, together with a fit to a Gaussian distribution. It is apparent that the distribution is substantially of Gaussian form at low amplitudes, but there are significant deviations near the tails. We only show in the figure the $x$ component of the acceleration field. The distribution for the other two components is virtually identical.

Figure 4 presents the results of higher statistical moments. Normalized cumulants have been introduced as follows:

$$
C_{m n}(t)=\frac{\left\langle\left\langle g(0)^{m} g(t)^{n}\right\rangle\right\rangle}{\left\langle\left\langle g^{2}\right\rangle\right\rangle^{(m+n) / 2}},
$$

where $\langle\langle\ldots\rangle\rangle$ is the standard cumulant. ${ }^{16}$ For a Gaussian process, all cumulants should be zero except for $C_{20}(t)$

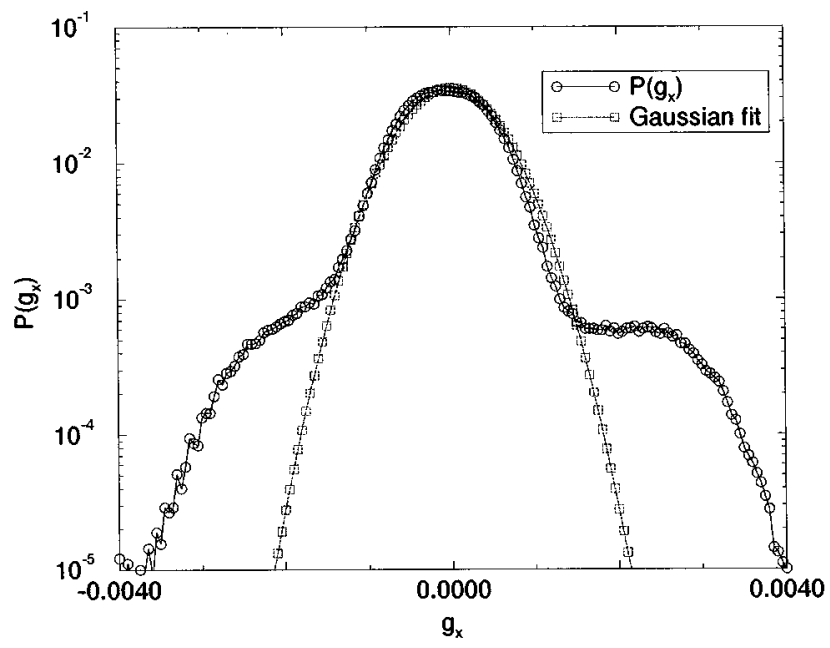

FIG. 3. Histogram of amplitudes of the residual acceleration along a particular direction, and a fit to a Gaussian distribution. The distribution is nearly Gaussian for small values of $g$, but deviates significantly near the wings. Values of the gravitational field intensity are given relative to $g_{E}$.

$=C_{02}(t)$ and $C_{11}(t)$. Also note that $C_{12}(0)=C_{21}(0)$ reduces to the standard definition of skewness of a distribution, and $C_{22}(0)$ to its kurtosis. Again, significant deviations from Gaussianity are found. Further analysis is needed to elucidate whether the deviations from Gaussianity in both Figs. 3 and 4 originate entirely from the deterministic component at 17 $\mathrm{Hz}$, or are a more intrinsic feature of the random components.

\section{COARSENING OF SOLID-LIQUID MIXTURES}

We consider in this section coarsening of a solid-liquid mixture being subjected to a fluctuating acceleration field of the type described in Sec. II. Such a study is relevant in connection with an experiment that will be conducted in microgravity in the near future. For purely diffusion controlled coarsening, larger precipitate particles grow at the expense of smaller ones by solute diffusion through the liquid matrix, so

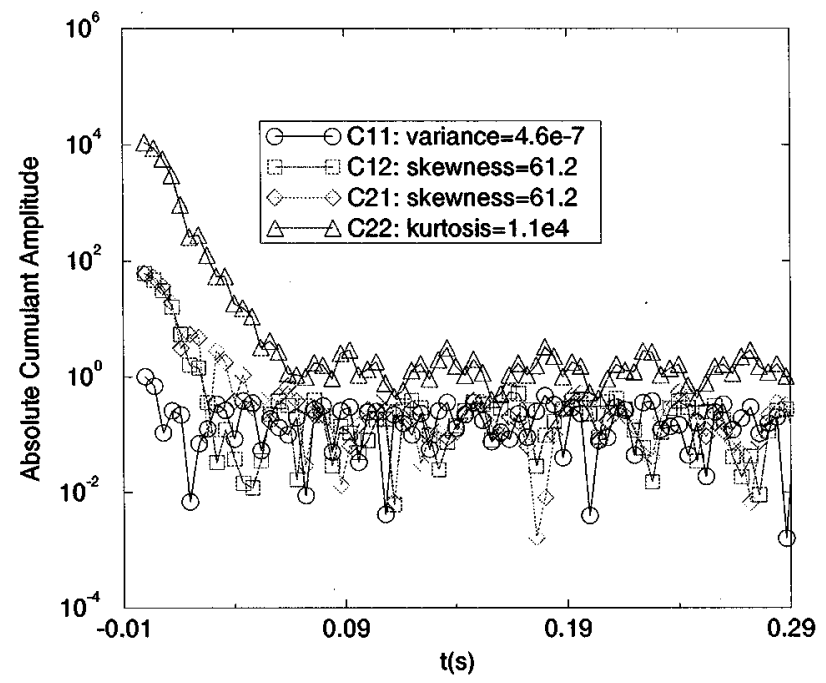

FIG. 4. Higher order moments of the distribution of the residual acceleration showing deviations from Gaussianity. 
as to minimize the overall free energy of the system. Under these conditions, the system is known to reach a statistical self-similar state in which the scaled distribution of particle radii is independent of time. ${ }^{17,18}$ Particle radii are scaled by any linear scale of the structure, such as, for example, the average particle size $R_{a v}(t)$. The average particle size follows a power law in time $R_{a v}=A(\phi) t^{n}{ }^{19}$ with $n=1 / 3$, and the amplitude $A$ being a function of the volume fraction of the precipitate phase $\phi$. The microgravity experiment will attempt a precise determination of the scaled distribution function, as well as the dependence of the amplitude of the growth law on the volume fraction of the precipitate phase. The absence of gravitationally induced sedimentation is expected to allow a careful quantitative study of these two important theoretical issues.

A residual acceleration field can produce a number of deleterious effects on otherwise purely diffusive controlled coarsening, which we address in this section. We focus here on two such effects: random motion of the suspended particles induced by the effective (random) buoyant force and the concomitant increase in the likelihood of particle coalescence, and additional convective mass transport in the fluid phase caused by g-jitter.

We first briefly review the motion of a single particle suspended in an incompressible fluid of different density, when the fluid is subjected to an effective acceleration field of the type described in Sec. II. This type of motion has also been termed inertial random walk, because of the similarity with Brownian motion. The difference, of course, is that the random motion of the particle is not induced by thermally induced collisions with the molecules of the fluid, but it results from an effective random buoyancy force acting on the particle. A qualitative analysis of this process was already given in Ref. 20.

Consider a spherical particle of radius $R$ and density $\rho_{p}$ submerged in an incompressible fluid of density $\rho_{f}$. If the fluid is enclosed by perfectly rigid boundaries, the buoyancy force acting on the submerged particle is $\vec{F}_{b}=\frac{4}{3} \pi\left(\rho_{p}\right.$ $\left.-\rho_{f}\right) R^{3} \vec{g}(t)$, where $\vec{g}(t)$ is the effective acceleration field. In the frame of reference co-moving with the container enclosing the fluid, $\vec{g}(t)$ is a body force, with intensity equal to the value of the acceleration of the container. For containers of reasonable size in a microgravity environment, $\vec{g}$ can be assumed to be spatially uniform. Viscous friction will act on the particle. Neglecting memory terms and corrections due to the finite size of the container, the viscous force is given by Stokes' formula $\vec{F}_{v}=-6 \pi \eta R \vec{v}$, where $\eta$ is the shear viscosity of the fluid, and $\vec{v}$ the velocity of the particle relative to the fluid at infinity. For simplicity, we consider in what follows a one dimensional case and write

$$
\ddot{x}+\gamma \dot{x}=\Delta \rho g(t),
$$

with $\gamma=9 \eta /\left(2 \rho_{p} R^{2}\right)$ and $\Delta \rho=\left(\rho_{p}-\rho_{f}\right) / \rho_{p}$. This is a standard Langevin equation for the particle displacement. At long times $(\gamma t \gg 1)$, the particle reaches a steady state in which the mean squared velocity is given by,

$$
\left\langle v^{2}\right\rangle_{\infty}=\frac{\Delta \rho^{2}\left\langle g^{2}\right\rangle\left(\gamma+\frac{1}{\tau}\right)}{\gamma\left[\left(\gamma+\frac{1}{\tau}\right)^{2}+\Omega^{2}\right]} .
$$

In the white noise limit, one has,

$$
\lim _{\tau \rightarrow 0}\left\langle v^{2}\right\rangle_{\infty}=\frac{\Delta \rho^{2}\left\langle g^{2}\right\rangle \tau}{\gamma}=\frac{\Delta \rho^{2} D}{\gamma},
$$

whereas in the monochromatic case

$$
\lim _{\tau \rightarrow \infty}\left\langle v^{2}\right\rangle_{\infty}=\frac{\Delta \rho^{2}\left\langle g^{2}\right\rangle}{\gamma^{2}+\Omega^{2}} .
$$

(An average over phases of the deterministic forces is assumed in the monochromatic noise limit, otherwise $\left\langle v^{2}\right\rangle_{\infty}$ would be an oscillatory quantity.) In the white noise limit, $\left\langle v^{2}\right\rangle_{\infty}$ is given by a fluctuation-dissipation relation since $\Delta \rho^{2} D$ is the intensity of the fluctuations, and $\gamma$ the intensity of the dissipation. In the monochromatic limit, however, $\left\langle v^{2}\right\rangle_{\infty} \propto 1 / \gamma^{2}$ (for low frequencies, $\Omega / \gamma \ll 1$ ). This is precisely the overdamped limit of Eq. (7). In all cases the mean squared value of the velocity saturates at a finite value at long times. In the monochromatic noise limit, this is the case even in the limit of small viscosity $\gamma \rightarrow 0$. In the white noise limit, on the other hand, viscosity is essential for saturation.

We turn next to the displacement of the particle. At long times $(\gamma t \gg 1)$, the motion of the particle is diffusive, with a diffusion coefficient given by ${ }^{21}$

$$
D_{e f f}=\int_{0}^{\infty}\langle v(0) v(t)\rangle d t .
$$

One finds

$$
D_{e f f}=\frac{\Delta \rho^{2}\left\langle g^{2}\right\rangle \lambda}{\gamma^{2}\left(\lambda^{2}+\Omega^{2}\right)} .
$$

As expected, the diffusion coefficient vanishes for $\tau \rightarrow \infty$.

For the monochromatic case, the displacement of a particle with $x(0)=0$ and $v(0)=0$ can be easily obtained. Square the deterministic solution for $g(t)=g_{0} \cos (\Omega t+\phi)$ and average over the phase $\phi$. With the identification $\left\langle g^{2}\right\rangle=g_{0}^{2} / 2$ and for $\gamma t \gg 1$, we obtain

$$
\begin{aligned}
\left\langle x^{2}(t)\right\rangle= & \frac{\Delta \rho^{2}\left\langle g^{2}\right\rangle}{\left(\gamma^{2}+\Omega^{2}\right)^{2}}\left[1+\left(\frac{\gamma}{\Omega}\right)^{2}+\left(\frac{\gamma}{\Omega}+\frac{\Omega}{\gamma}\right)^{2}-2\left(\frac{\gamma}{\Omega}\right.\right. \\
& \left.\left.+\frac{\Omega}{\gamma}\right)\left(\frac{\gamma}{\Omega} \cos \Omega t+\sin \Omega t\right)\right] .
\end{aligned}
$$

In this case the mean square displacement of the particle is bounded. For the particular case of $\gamma=0$, Eq. (13) is not valid and the mean square displacement of the particle may be unbounded. Since the correlation time of the noise diverges, $\left\langle x^{2}(t)\right\rangle$ retains an oscillatory contribution originating from the initial condition. We also note that, in the monochromatic limit, the system is no longer ergodic.

In order to obtain numerical estimates we consider experimental parameters for a solid-liquid mixture of Sn-rich particles in a Pb-Sn eutectic liquid, the system that will be used in the microgravity experiment. ${ }^{22,23}$ The density of the 
precipitating solid phase is $\rho_{p}=7.088 \mathrm{~g} / \mathrm{cm}^{3}$, whereas that of the liquid is $\rho_{l}=8.074 \mathrm{~g} / \mathrm{cm}^{3}$. The kinematic viscosity of the liquid is $\nu=2.48 \times 10^{-3} \mathrm{~cm}^{2} / \mathrm{s}$, and the solute diffusivity is $D_{s}=5.6 \times 10^{-6} \mathrm{~cm}^{2} / \mathrm{s}$. It is anticipated that coarsening will be studied for a period of 5 hours, with an average particle size at the end of that period of $R_{a v} \simeq 7$ $\times 10^{-3} \mathrm{~cm}=70 \mu \mathrm{m}$. Given the size of the particles and the small values of the residual gravitational field, inertial effects will be completely negligible. In what follows, we focus almost exclusively on Stokesian dynamics for the suspended particles. ${ }^{24}$ In addition, the solution is not mono-disperse, but rather a scale invariant particle size distribution evolves dynamically, ${ }^{17}$ with particles sizes ranging from 0 to $\approx 1.5 R_{a v}$. In all the estimates that follow, the average size at the end of the 5 hours is used, an overestimate for most of the duration of the experiment, and a slight underestimate at the latest times. As will be seen below, a factor of two in $R_{a v}$ would not modify our conclusions.

For the case of monochromatic noise (fixed frequency and random phase), the average quadratic displacement of each particle remains bounded, and is given by Eq. (13). For the parameters of the fluid given $\gamma=260 \mathrm{~s}^{-1}$, and by using the amplitude of the $17 \mathrm{~Hz}$ component of the power spectrum in Sec. II $\left(\Omega=2 \pi 17 \mathrm{~s}^{-1},\left\langle g^{2}\right\rangle=1.27 \times 10^{-7} g_{E}^{2}\right)$, we find that $\max \left\{\left\langle x^{2}\right\rangle\right\} \approx 10^{-8} \mathrm{~cm}^{2}$, and hence negligible. At the other extreme, we find that for white noise (multiplying by a factor of 3 the expression for the effective diffusion coefficient corresponding to diffusion in three dimensional space), the mean squared displacement after five hours is $\left\langle x^{2}\right\rangle(t=5 \mathrm{hr})=8.85 \times 10^{-6} \mathrm{~cm}^{2}$ or $\sqrt{\left\langle x^{2}\right\rangle} \simeq 30 \mu \mathrm{m}$. Clearly the average square displacement induced by the white noise component of the residual acceleration field is much larger than that induced by the monochromatic component, but it is still about one-half of the average particle size. Therefore random motion of particles induced by g-jitter will not lead to significant coalescence during this time period. As a reference, we quote the average squared displacement induced by thermal Brownian motion: $\left\langle x^{2}\right\rangle=k_{B} T t / 3 \pi R_{a v} \eta$ or $\sqrt{\left\langle x^{2}\right\rangle} \simeq 39 \mu \mathrm{m}$, for the same time span and taking $R_{a v}=70$ $\mu \mathrm{m}$. Both effects are therefore expected to be of the same order of magnitude during the experiment.

We next turn to hydrodynamic interactions between pairs of spherical particles. The relative displacement $\vec{r}$ of particle 2 with respect to particle 1 immersed in an incompressible fluid satisfies ${ }^{25,26}$

$$
\frac{d \vec{r}}{d t}=\left(\boldsymbol{\omega}_{21}-\boldsymbol{\omega}_{11}\right) \cdot \vec{F}_{1}+\left(\boldsymbol{\omega}_{22}-\boldsymbol{\omega}_{21}\right) \cdot \vec{F}_{2}
$$

where $\vec{F}_{i}$ is the force acting on the $i$-th particle, and $\boldsymbol{\omega}_{i j}$ are hydrodynamic mobility tensors, given, e.g., in Refs. 25 and 26. After some straightforward algebra, the leading contribution at distances large compared to the particle radii is given by

$$
\begin{aligned}
\frac{d \vec{r}}{d t}= & \frac{2\left(\rho_{p}-\rho_{f}\right)}{9 \mu}\left(R_{2}^{2}-R_{1}^{2}\right) \vec{g}(t) \\
& +\frac{\left(\rho_{p}-\rho_{f}\right)\left(R_{1}^{3}-R_{2}^{3}\right)}{3 \mu} \frac{1}{r}\left[\frac{\vec{r} \vec{r}}{r^{2}}+\frac{1}{2}\left(\mathscr{T}-\frac{\vec{r} \vec{r}}{r^{2}}\right)\right] \cdot \vec{g}(t),
\end{aligned}
$$

where $\mathscr{T}$ is the identity tensor. The first term in the right hand side describes the relative motion of two independent particles of different size, and therefore its magnitude has already been estimated above. Both the longitudinal and transverse components of the second term in the right hand side of Eq. (15) are of the form

$$
\frac{d r}{d t}=\frac{A}{r} g(t),
$$

where, for the longitudinal component, $A=\left(\rho_{p}-\rho_{f}\right)\left(R_{1}^{3}\right.$ $\left.-R_{2}^{3}\right) / 3 \mu$.

Consider an initial inter-particle separation $r_{0} \gg R_{i}$. In this case, and for times shorter than the average time needed for the two particles to coalesce, the quantity $y=r^{2} / 2 A$ is a Wiener process if $g(t)$ is Gaussian and white, and therefore the conditional probability for $r$ is

$$
P\left(r, t \mid r_{0}, t_{0}\right)=\frac{r}{|A| \sqrt{4 \pi D\left(t-t_{0}\right)}} e^{-\left(r^{2}-r_{0}^{2}\right)^{2} / 16 D A^{2}\left(t-t_{0}\right)} .
$$

The ensemble average of $r,\langle r\rangle$, can be computed analytically:

$$
\begin{aligned}
& \langle r\rangle=\sqrt{\frac{|A|}{4}}\left[2 D\left(t-t_{0}\right)\right] e^{-r_{0}^{4} / 32 A^{2} D\left(t-t_{0}\right)} \\
& \times D_{-3 / 2}\left(-\frac{r_{0}^{2}}{2|A| \sqrt{2 D\left(t-t_{0}\right)}}\right),
\end{aligned}
$$

where $D_{p}(z)$ is a parabolic cylinder function ${ }^{27}$ (formula 9.240). For short times, the asymptotic form of $D_{p}(z)$ for large $z$ allows the computation of $\langle r\rangle$

$$
\langle r\rangle=r_{0}\left(1-\frac{A^{2} D\left(t-t_{0}\right)}{r_{0}^{4}}\right),
$$

which decreases in time regardless of the sign of $A$. Therefore g-jitter induces an effective hydrodynamic attraction between pairs of particles. However, for the experimental values given above, and taking $R_{1}=1.5 R_{a v}$ and $R_{2}=0.5 R_{a v}$, $A=1.8 \times 10^{-5} \mathrm{~cm} \mathrm{~s}$. If $r_{0} \simeq 200 \mu \mathrm{m}$, then after 5 hours the inter-particle separation would have decreased by approximately $7 \mu \mathrm{m}$, and therefore would be small compared to particle radii.

The attractive interaction is not confined to short times, but it arises directly from the $1 / r$ dependence in Eq. (16). By taking the average of Eq. (16), using the Furutsu-Novikov theorem ${ }^{28}$ and the fact that the noise is Gaussian and white, one finds

$$
\frac{d\langle r\rangle}{d t}=A D\left\langle\frac{\delta 1 / r(t)}{\delta g(t)}\right\rangle,
$$


where $\delta / \delta g(t)$ stands for functional derivative with respect to $g$. Directly from Eq. (16), we find that $\delta(1 / r(t)) /$ $\delta g(t)=-A / r^{3}$, and therefore,

$$
\frac{d\langle r\rangle}{d t}=-A^{2} D\left\langle\frac{1}{r^{3}}\right\rangle
$$

identical to Eq. (19) with $1 / r_{0}^{3}$ replaced by $\left\langle 1 / r^{3}\right\rangle$. Since $r$ is a positive quantity, $d\langle r\rangle / d t<0$ for all values of $r$. It is also interesting to note that the effective attractive interaction is not confined to the term proportional to $1 / r$ in the hydrodynamic mobility, but that attractive contributions arise from higher powers of $1 / r$ as well. In fact, this attraction is generic for overdamped motion and multiplicative noise provided that the mobility is a decaying function of the inter-particle separation. ${ }^{29}$

The question naturally arises as to the behavior of pairs of particles near contact, or of particles near a solid wall. In either case, lubrication theory allows the calculation of the mobility tensor. The longitudinal component vanishes linearly with inter-particle distance whereas the transverse component becomes non-analytic (diverges logarithmically at short distances). ${ }^{26}$ In both cases, the mobility increases with inter-particle separation leading to an average repulsion $(d\langle r\rangle / d t>0)$ following the same arguments given above.

Estimating the effect of g-jitter on convective mass transport in the fluid phase and therefore on coarsening kinetics is far more complex, and we will not attempt a complete solution here. We show below that the order of magnitude of this contribution to coarsening is also small compared to diffusive mass transport, and therefore a detailed calculation is not necessary. However, and in order to motivate the analysis that follows, let us define an effective Peclet number as $\mathrm{Pe}=\sqrt{\left\langle u^{2}\right\rangle} R / D_{s}$, where $\vec{u}$ is the characteristic velocity of the fluid. (The velocity $\vec{u}$ is of the order of the velocity of the particles because the motion of two-phase interfaces due to phase change is small in the time scale of change of $g(t)$.) We find for monochromatic noise (Eq. (10)) that $\left\langle u^{2}\right\rangle=3.48 \times 10^{-8} \mathrm{~cm}^{2} / \mathrm{s}^{2}$ or $\mathrm{Pe}=0.23$. For white noise, on the other hand, according to Eq. (9), $\left\langle u^{2}\right\rangle=6.39$ $\times 10^{-8} \mathrm{~cm}^{2} / \mathrm{s}^{2}$, or $\mathrm{Pe}=0.32$. Therefore it would appear that convective transport of mass is not negligible in front of diffusive transport.

Such a calculation, however, overestimates convective transport. Since the system is statistically uniform and the effective acceleration field averages to zero, the average velocity of the fluid has to be zero. Hence no overall convective motion would result in the (longer) time scale over which diffusive transport occurs, once the fluctuating component is averaged over times much larger than the correlation time of the noise. Since $\langle\vec{u}\rangle=0$ but $\left\langle u^{2}\right\rangle \neq 0$, the lowest order contribution to transport due to the motion of a fluid element is diffusive. ${ }^{30}$ The calculation of this effective diffusivity can be carried out in a mean field approximation. Consider a single solid particle immersed in the fluid phase, so that the presence of the remaining particles can be subsumed in a far field composition $c_{\infty},{ }^{17}$ and a far field velocity $\vec{u}_{\infty}$, both to be determined self-consistently for a given particle distribution. The cut-off distance is typically of the order of the inter-particle separation.

Fluctuations in $\vec{u}_{\infty}$ due to the motion of the ensemble of particles (again, fast compared to coarsening times) lead to an effective increase in diffusive mass transport, and hence to an increased diffusivity. For a quiescent system, the average velocity of the fluid $\vec{u}_{a v}$ and the average velocity of the particles $\vec{v}_{a v}$ are related by

$$
\phi \vec{v}_{a v}+(1-\phi) \vec{u}_{a v}=0,
$$

where $\phi$ is the volume fraction of the system, which we assume to be small. The subindex $a v$ indicates an average over the particle distribution at fixed time. In mean field, we take $\vec{u}_{\infty}=\vec{u}_{a v}$, and also

$$
\vec{v}_{a v}=\frac{2 R_{a v}^{2}\left(\rho_{p}-\rho_{f}\right) \vec{g}(t)}{9 \eta}+\mathscr{O}(\phi)
$$

with hydrodynamic interactions contributing to $\mathscr{O}(\phi)$. Therefore,

$$
\vec{u}_{\infty}=-\frac{2 \phi R_{a v}^{2}\left(\rho_{p}-\rho_{f}\right) \vec{g}(t)}{9 \eta}+\mathcal{O}\left(\phi^{2}\right) .
$$

The far field effective diffusivity is then ${ }^{21,31}$

$D_{e f f}=D_{s}+\frac{1}{3} \int_{0}^{\infty}\left\langle\vec{u}_{\infty}(t) \cdot \vec{u}_{\infty}\left(t+t^{\prime}\right)\right\rangle d t^{\prime}=D_{s}+\frac{\Delta \rho^{2} \phi^{2} D}{\gamma_{a v}^{2}}$

in the white noise limit. For the parameters of the experiment given above

$$
\frac{\Delta \rho^{2} \phi^{2} D}{\gamma_{a v}^{2} D_{s}} \ll 1,
$$

and therefore g-jitter induced mass transport by diffusion is negligible compared to molecular diffusion.

In summary, even though anticipated Peclet numbers based on the scale of the flow are of order unity, mass transport due to convection is expected to be negligible during the solid-liquid coarsening experiment. Since the time scale of acceleration variations is short compared to coarsening times, and leads to zero average velocity, the contribution from g-jitter to mass transport is diffusive and leads to a very small correction to the solute diffusivity. We note, however, that the correction is proportional to $1 / \gamma_{a v}^{2} \propto R_{a v}^{4}$ and hence it increases quickly with the average particle size of the precipitate phase. Therefore, either under different experimental conditions, or in the strict asymptotic limit of very long times (and hence large $R_{a v}$ ), transport due to transient accelerations would dominate molecular diffusion leading to a different asymptotic growth law for the average particle size.

\section{ACKNOWLEDGMENTS}

We are indebted to Richard DeLombard and the PIMS project at Lewis Research Center for providing us with the SAMS accelerometer data used in this paper, and also to Peter Voorhees for information on the experiment on "Coarsening in Solid-Liquid Mixtures." This work is supported by the Microgravity Science and Applications Divi- 
sion of the NASA under Contract No. NAG3-1284, and also in part by the Supercomputer Computations Research Institute, which is partially funded by the U.S. Department of Energy, Contract No. DE-FC05-85ER25000. J.C. is also supported by the Dirección General de Investigación Científica y Técnica, Contract No. PB93-0769.

${ }^{1}$ Fluid Sciences and Materials Sciences in Space, edited by H. Walter (Springer Verlag, New York, 1987).

${ }^{2}$ Low-Gravity Fluid Dynamics and Transport Phenomena, Vol. 130 of Progress in Aeronautics and Astronautics, edited by J. Koster and R. Sani (AIAA, Washington, 1990).

${ }^{3}$ Microgravity Fluid Mechanics, edited by H. Rath (Springer Verlag, New York, 1992).

${ }^{4}$ B. Antar and V. Nuotio-Antar, Fundamentals of Low Gravity Fluid Dynamics and Heat Transfer (CRC, Boca Raton, FL, 1993).

${ }^{5} \mathrm{G}$. Martin, C. Baugher, and F. Henderson, "Summary report of mission acceleration measurements, STS-50," NASA Technical report, Acceleration Characterization and Analysis Project (ACAP) (unpublished).

${ }^{6}$ J. Alexander, "Low-gravity experiment sensitivity to residual acceleration: a review," Micrograv. Sci. Technol. 3, 52 (1990).

${ }^{7}$ Y. Kamotani, A. Prasad, and S. Ostrach, "Thermal convection in an enclosure due to vibrations aboard a spacecraft," AIAA J. 19, 511 (1981).

${ }^{8} \mathrm{D}$. Jacqmin, "Stability of an oscillated fluid with a uniform density gradient," J. Fluid Mech. 219, 449 (1990).

${ }^{9}$ J. Alexander, J. Ouazzani, and F. Rosenberger, "Analysis of the low gravity tolerance of Bridgman-Stockbarger crystal growth," J. Crystal Growth 113, 21 (1991).

${ }^{10}$ T. C. Jue and B. Ramaswamy, "Natural convection with thermocapillary and gravity mondulation effects in low-gravity environments," J. Spacecraft Rockets 29, 856 (1992).

${ }^{11}$ A. Farooq and G. Homsy, "Streaming flows due to g-jitter-induced natural convection,', J. Fluid Mech. 271, 351 (1994).

${ }^{12} \mathrm{~W}$. Zhang, J. Casademunt, and J. Viñals, "Study of the parametric oscillator driven by narrow band noise to model the response of a fluid surface to time-dependent accelerations," Phys. Fluids A 5, 3147 (1993).

${ }^{13}$ J. Thomson, J. Casademunt, and J. Viñals, "Cavity flow induced by a fluctuating acceleration field,' Phys. Fluids 7, 292 (1995).

${ }^{14} \mathrm{~B}$. Matisak, M. Rogers, and J. Alexander, in Proceedings of the 32nd Aerospace Sciences Meeting (AIAA, Washington, 1994), paper AIAA 940434.

${ }^{15}$ M. Priestley, Spectral Analysis and Time Series. Volume I, Univariate Series, Probability and Mathematical Statistics Series (Academic, New York, 1981)

${ }^{16}$ S.-K. Ma, Statistical Mechanics (World Scientific, Singapore, 1985), pp. 203-205.
${ }^{17}$ J. D. Gunton, M. San Miguel, and P. S. Sahni, in Kinetics of first order phase transitions, Vol. 8 of Phase Transitions and Critical Phenomena, edited by C. Domb and J. Lebowitz (Academic, London, 1983).

${ }^{18} \mathrm{~W}$. W. Mullins and J. Viñals, "Self-similarity and growth kinetics driven by surface free energy reduction," Acta Metall. 37, 991 (1989).

${ }^{19}$ I. Lifshitz and V. Slyozov, J. Phys. Chem. Solids 19, 35 (1961)

${ }^{20}$ L. Regel, A. Vedernikov, R. Ilinski, and I. Melikhov, in Proceedings of the Sixth European Symposium on Materials Sciences under Microgravity Conditions (European Space Agency, Paris, 1987), ESA Publication SP256.

${ }^{21}$ R. Kubo, M. Toda, and N. Hashitsume, Statistical Physics II, Nonequilibrium Statistical Mechanics (Springer-Verlag, New York, 1985).

${ }^{22}$ S. Hardy and P. Voorhees, Met. Trans. A 19, 2713 (1988).

${ }^{23}$ S. Hardy, G. McFadden, S. Coriell, P. Voorhees, and R. Sekerka, "Measurement and analysis of grain boundary grooving by volume diffusion," J. Crystal Growth 114, 467 (1991).

${ }^{24}$ Inertial effects must be included for the case of white noise, or, in general, for colored noise when the correlation time $\tau$ is smaller or of the order of the viscous damping time $1 / \gamma$ (see Eq. (8)). In the case considered, $1 / \gamma=1 / 260 \mathrm{~s}$, smaller than the sampling period of the acceleration data. Second, Stokes dynamics also fails at distances longer than the momentum diffusion length $l_{\nu}$. In the case at hand $l_{\nu}=\nu / u \simeq(2.48$ $\left.\times 10^{-3} \mathrm{~cm}^{2} / \mathrm{s}\right) /\left(10^{-4} \mathrm{~cm} / \mathrm{s}\right)=24.8 \mathrm{~cm}$ larger than the dimensions of the container. Hence the motion of the fluid surrounding the particles is overdamped, and satisfies the equation for Stokes flow driven by the instantaneous velocity of the particles.

${ }^{25} \mathrm{G}$. Batchelor, "Brownian diffusion of particles with hydrodynamic interaction," J. Fluid Mech. 75, 1 (1976).

${ }^{26}$ W. Russel, D. Saville, and W. Schowalter, Colloidal Dispersions (Cambridge U. P., New York, 1989).

${ }^{27}$ I. Gradshteyn and I. Ryzhik, Tables of Integrals, Series and Products (Academic, New York, 1980)

${ }^{28} \mathrm{P}$. Hanggi, in Stochastic Processes Applied to Physics, edited by L. Pesquera and M. Rodriguez (World Scientific, Singapore, 1985), p. 69.

${ }^{29}$ It is important to emphasize the relevance of overdamped motion to the results presented. If the correlation time of the fluctuating force is smaller than the viscous decay time (as is the case in thermal Brownian motion), the fluctuating force enters additively in the equations of motion for the particles and the effects discussed do not arise. In the present case, however, the viscous decay time is $1 / 260 \mathrm{~s}$ much smaller than the correlation time of the effective acceleration field. In this limit, the fluctuating component enters multiplicatively and leads to an average attraction at long distances (but still shorter than the visous decay length), and repulsion at short distances.

${ }^{30}$ There is no large scale (low wave number) motion as in the case of turbulent flow. The Reynolds number is typically much smaller than unity.

${ }^{31}$ J. Boon and S. Yip, Molecular Hydrodynamics (Dover, New York, 1991). 